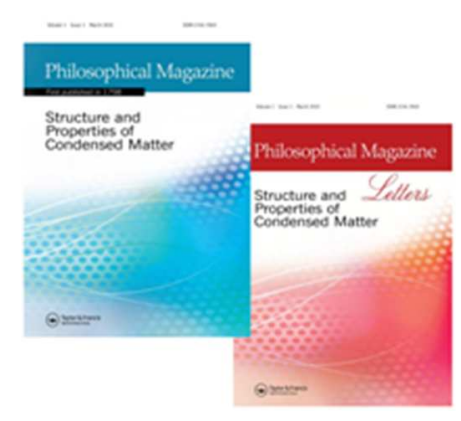

\title{
Incipient plasticity of single Crystal Tantalum as a function of temperature and orientation
}

\begin{tabular}{|c|c|}
\hline Journal: & Philosophical Magazine \& Philosophical Magazine Letters \\
\hline Manuscript ID: & TPHM-13-Nov-0467.R1 \\
\hline Journal Selection: & Philosophical Magazine \\
\hline Date Submitted by the Author: & 17-May-2014 \\
\hline Complete List of Authors: & $\begin{array}{l}\text { Franke, Oliver; University of Southern California, Aerospace and } \\
\text { Mechanical Engineering } \\
\text { Alcala, Jorge; Universitat Politècnica de Catalunya. BarcelonaTech, } \\
\text { Zhichao, Duan; University of Southern California, Aerospace and } \\
\text { Mechanical Engineering } \\
\text { Biener, Juergen; Lawrence Livermore National Laboratory, } \\
\text { Biener, Monika; Lawrence Livermore National Laboratory, } \\
\text { Dalmau, Roger; Universitat Politècnica de Catalunya. BarcelonaTech, } \\
\text { Hodge, Andrea; University of Southern California, AME }\end{array}$ \\
\hline Keywords: & nanomechanics, tantalum, finite-element modelling, nanoindentation \\
\hline Keywords (user supplied): & \\
\hline
\end{tabular}


Incipient plasticity of single Crystal Tantalum as a function of temperature and orientation

\title{
O. Franke ${ }^{1}$, J. Alcalá ${ }^{2}$, R. Dalmau ${ }^{2 \S}$, Zhi Chao Duan ${ }^{1}$, J. Biener ${ }^{1,3}$, M.M. Biener ${ }^{3}$,
} A.M. Hodge ${ }^{1, *}$

${ }^{1}$ Department of Aerospace and Mechanical Engineering, University of Southern California, Los Angeles

${ }^{2}$ Universitat Politècnica de Catalunya. BarcelonaTech, Barcelona Spain

${ }^{3}$ Lawrence Livermore National Laboratory, Livermore California

${ }^{*}$ corresponding author

$\S$ now at: New Technologies Research Centre, University of West Bohemia in Pilsen, Plzeň, Czech Republic

\begin{abstract}
The nanocontact plastic behavior of single-crystalline Ta (100), Ta (110) and Ta (111) was studied as a function of temperature and indentation rate. Tantalum, a representative body centered cubic (BCC) metal, reveals a unique deformation behavior dominated by twinning and the generation of stacking faults. Experiments performed at room temperature exhibit a single pop-in event, while at $200^{\circ} \mathrm{C}$, above the critical temperature, a transition to multiple pop-ins was observed. The experimental results are discussed in respect to the orientation as well as temperature and correlated to the defect structures using both anisotropic finite element and MD simulations. The serrated flow observed at $200^{\circ} \mathrm{C}$ is related to differences in the quasi-elastic reloading originating from changes in the defect mechanism.
\end{abstract}

\section{Keywords}

A: Physical phenomena: Dislocation, Twinning; B: Problem description: elastic-plastic material; C: Methods: finite elements, mechanical testing 


\section{Introduction}

The transition from elastic to the incipient plastic regime measured through nanoindentation experiments of different material's surfaces has been a topic of high interest in the past years [1-13]. The strength of this technique lies in its ability to probe ultra-small and therefore virtually "defect-free" sample volumes. In well-prepared surfaces of single crystals, this leads to the appearance of discontinuities in the loaddisplacement curves that are generally explained by homogeneous nucleation of dislocations loops in the area of maximum stress underneath the indenter [14-18]. However, atomistic simulations showed that the homogeneous dislocation nucleation assumption might not be true and that dislocations can in fact nucleate heterogeneously [17] or by completely different mechanisms such as twinning [19]. Compared to face centered cubic (FCC) systems, where the correlation between incipient plasticity and dislocation motion has been well established [5, 6, 8, 14-18, 20-22]. BCC systems are much more complex and, despite several reports [11-13, 23-29], there are still many unanswered questions regarding our understanding of BCC-plasticity in nanocontacts encountered in indentation.

Most recent experimental studies focusing on the defect evolution in BCC-materials at small scales so far were carried out on nanopillars [23, 24, 26, 28, 29] with a few studies 
using nanoindentation [20, 27]. A recent simulation study by Li et al. defined a critical pop-in load in nanoindentation experiments based on the Schmid factor for the different crystallographic planes, which could then be considered as the load required for homogeneous dislocation nucleation using similar arguments as those employed for FCC metals. Their study also illustrated the influence of crystal anisotropy, indenter tip geometry and operative slip systems in shifting the locus of the maximum shear stress from the center of the indentation as a function of indenter geometry, elastic anisotropy and operative slip systems [30]. Schneider et al. showed that both orientation and the ratio of the test temperature to the critical temperature play an important role in the deformation behavior [29]. While the orientation mostly affects the stress on the slip systems, the ratio between test and critical temperature is a measure for the screw dislocation mobility, which is a limiting factor in the deformation of BCC metals.

Even though there is a significant amount of available slip systems in BCC (48 total) the slip direction $(<111>$ ) and the slip planes $((110),(123)$ and (112)) are significantly less densely packed than in FCC. This is one of the reasons why nonconservative dislocation motion such as cross-slip of screws plays a more important role in the plasticity of BCCs. The core of screw dislocations in BCC-Ta is also found to degenerate into different planes of a common zone axis which significantly reduces the mobility of screw as compared to edge dislocation segments [31,32]. The lack of local surface energy minima in any slip plane also prevents the well-known splitting of full dislocations into partial dislocations separated by ribbons of stacking faults as found in pure FCC metals. To adapt to this distinctive behavior, Dorn and Mukharjee modified the classical Peierl's potential with an effective stress that permits cross slip of screw 
dislocations [33]. At the critical temperature screw dislocations become thermally activated and their mobility reaches that of edge dislocations. In nanoindentation experiments, this leads to a transition to a serrated flow behavior that is characterized by multiple pop-ins and smaller displacement bursts (e.g. [20, 27]). Similar approaches were adopted by Nemat Nasser et al. in setting continuum crystal plasticity models for BCC metals [34] and in the work by Naka et al for the thermodynamical treatment of temperature effects in enhancing mobility of screw dislocations through mechanisms such as kink-pair formation [35]. Accordingly, screw dislocations are found to glide through thermally assisted formation of local edge segments whose motion is parallel to the dislocation line of the preexisting screw [36]. This mechanism is also important for cross-slip effects in BCCs [37].

Two different approaches can be used to test the effect of temperature on incipient plasticity of BCC metals: One can test different materials with different critical temperatures at room temperature, thus effectively testing at different homologous temperatures [29]. While this approach is experimentally straightforward, the interpretation becomes complicated by simultaneously changing elastic and plastic properties, including different screw dislocation core arrangements. In this manuscript, in order to avoid this complication, the tests are performed on only one material, Ta, and the test temperature is increased up to $200^{\circ} \mathrm{C}$, which is above the critical temperature of $\mathrm{Ta}$ $\left(177^{\circ} \mathrm{C}\right)[20,27]$. The experiments were accompanied by finite element simulations that account for anisotropic elasticity to evaluate the resolved shear stresses in all slip systems under fixed (applied) indentation loading conditions, as well as with large-scale 
molecular dynamics simulations to gain additional insights on the underlying deformation mechanisms and their orientation and temperature dependency [10].

\section{Experimental Setup}

Low index Tantalum single crystals, $\mathrm{Ta}(100), \mathrm{Ta}(110)$ and $\mathrm{Ta}(111)$ were prepared by a combination of electrochemical and mechanical polishing, resulting in rms surface roughness values below $1 \mathrm{~nm}$ [Surface Preparation Laboratory, The Netherlands]. The crystallographic orientation of the samples was determined prior to cutting by Laue diffraction. All nanoindentation experiments were performed in the load-controlled mode using a commercial Hysitron Triboindenter (MN,USA) system. A Berkovich tip with a radius of curvature of $\sim 190 \mathrm{~nm}$ was used to obtain quantitative load-displacement data, and a spherical tip with a tip radius of $\sim 1 \mu \mathrm{m}$ was used to study material flow under the indenter . Different loading rates of 1000 and $5000 \mu \mathrm{N} / \mathrm{s}$ were chosen both at room temperature and $200{ }^{\circ} \mathrm{C}$. For nanoindentation experiments at $200{ }^{\circ} \mathrm{C}$, the testing protocol described in an earlier paper $[20,27,38]$ was followed. The indents were imaged for both temperatures. The oxide structure and thickness were measured following the procedure described elsewhere [27].

Anisotropic elasticity finite element FE simulations were performed where the following relation is assumed to hold between the stresses and the strains

$\nabla$

$\tau=\mathbf{C}: \mathbf{D}$

where $\mathrm{C}$ is the elasticity matrix with $\mathrm{C}_{11}=256 \mathrm{GPa}, \mathrm{C}_{12}=166 \mathrm{GPa}$ and $\mathrm{C}_{44}=79 \mathrm{GPa}$ for Ta crystals [39]. (Although not given here for the sake of brevity, complementary FE simulations were also performed by accounting for the increase in $\mathrm{C}_{\mathrm{ij}}$ due to the level of 
confining pressure underneath the indent). All computations were performed by accounting for large strains and rotations, where the stresses and strains are computed in the deformed state. Notice that the above Eq. (1) employs the conventional treatment of large deformations where the Cauchy stress tensor and Green strain tensor are substituted by the Jaumann rate of the Kirchoff stress tensor $\stackrel{\nabla}{\tau}$ and rate of deformation tensor $\mathbf{D}$, respectively. Consideration of large strains was crucial in the present investigation because defect nucleation only occurs at large penetrations of the spherical tip where significant rotation of the material axes has already occurred. The outputs from the FE simulations were the P-h curves and the active shear stress in all of the 48 slip systems as found by projecting the stress tensor $\stackrel{\nabla}{\tau}$ for all material points underneath the surface in each of the 48 slip systems. The employed FE meshes are the same as those described in $[40]$.

\section{$\underline{\text { 3. Room temperature behavior }}$}

\subsection{Instrumented indentation response}

In room temperature experiments, all three orientations show very similar loaddisplacement curves with a single pop-in event indicating the onset of plasticity (Fig 1ab). The pop-in displacements are relatively large, typically several tens of nanometers, indicating the involvement of a significant number of dislocations.

As a first attempt to understand incipient plasticity, the influence of varying the sample orientation in relation of the surface normal to the slip planes are shown in table 1 . The contact can be considered Hertzian until the initial yielding shows as a discontinuity in the load-displacement curve since the displacement at which the initial pop-in occurs is in 
the order of $10-20 \%$ of the tip radius. The tip can thus be considered spherical for this approximation. Furthermore, even though the stress state underneath the indenter is more complex than in a regular uniaxial tensile or compression test, the highest stress is expected directly underneath the center of the indenter (parallel to the surface normal) at a distance $\mathrm{z}=0.48 \mathrm{a}$ (where $\mathrm{a}$ is the contact radius). Therefore, the predicted location for the highest shear stress is also the location where the initial yielding is expected to occur in compression along the loading direction. However, from Table 1, one would expect the lowest pop-in load to be found in the (100) crystal followed by (110) and then (111). Yet, the observed experimental results shown in Figure 1 displayed the opposite trend. Obviously a purely geometrical analysis (table 1) of the problem falls short of being able to explain the complex stress states and the resulting yield phenomena observed in nanoindentation. To develop a better understanding of nanocontact behavior in BCC materials, a series of simulations and elevated temperature experiments are implemented in order to fully develop an understanding of nanocontact behavior in $\mathrm{BCC}$ materials.

Table 1: Orientation relationships of surface normal and slip planes

\begin{tabular}{|c|c|c|c|c|}
\hline $\begin{array}{c}\text { Single crystal } \\
\text { orientation }\end{array}$ & $\begin{array}{c}\text { Angle surface } \\
\text { normal to [111] slip } \\
\text { direction }\end{array}$ & $\begin{array}{c}\text { Angle surface } \\
\text { normal to (110) slip } \\
\text { plane }\end{array}$ & $\begin{array}{c}\text { Angle surface } \\
\text { normal to (123) slip } \\
\text { plane }\end{array}$ & $\begin{array}{c}\text { Angle surface } \\
\text { normal to (112) slip } \\
\text { plane }\end{array}$ \\
\hline$(100)$ & $54.7^{\circ}$ & $45^{\circ}$ & $74.5^{\circ}$ & $65.9^{\circ}$ \\
\hline$(110)$ & $35.3^{\circ}$ & $0^{\circ}$ & $55.5^{\circ}$ & $54.7^{\circ}$ \\
\hline$(111)$ & $0^{\circ}$ & $35.3^{\circ}$ & $22.2^{\circ}$ & $19.5^{\circ}$ \\
\hline
\end{tabular}

\subsection{Critical stresses for defect nucleation}

In order to further explore the observed pop-in behavior, one must first look at the specific nucleation events for a given crystal orientation. In a previous publication, a 
comprehensive MD study demonstrated that the incipient contact plasticity in Ta is ruled by the nucleation of planar defects such as twins and stacking faults and that the defect nucleation processes are strongly dependent upon the crystalline orientation [10].

Therefore, a full 3D analysis of the stress fields for all the 48 slip systems in Ta was performed taking into account the specific nucleation events revealed by the MD simulations. The initial FE simulations showed that there are many different slip systems that share visually identical stress fields in terms of geometry and magnitude. So even though there are 48 slip systems, there are only 6 visually distinguishable resolved shear stress fields for (001) oriented specimen, 10 for (110) and 12 for (111).

By comparing the location of the nucleated defects in (001) and (011) indentations, one can establish a clear correlation between the locations of the maximum shear stresses for the most critical $\{011\}<0-11>$ and $\{112\}<11-1>$ slip systems and those where SF and twins nucleate, respectively [10]. This shows that defect nucleation is indeed driven by shear. The FE simulations (Fig. 2a-c) show that the most active slip system in the three surface orientations is found to be of the $\{211\}$ family. In these most active slip systems, it is also found that (011) indentations exhibit $22 \%$ greater maximum resolved shear stresses than (001) indentations, which in turn exhibit $8 \%$ greater maximum shear stresses than (111) indentations. Thus the 3D analysis of the stress fields suggests that the smallest pop-in loads should be encountered for the (001) surface.

However, those results are in contrast with experimental observations, thus additional analysis of the specific defect types and underlying tensional conditions are required in order to gain an accurate comprehension of the micromechanics of the pop-in phenomenon. For example the FE simulations show that stacking faults nucleate at a 
critical shear stress of $10.3 \mathrm{GPa}$ for (001) indentations while the value underlying twin nucleation is $9.7 \mathrm{GPa}$ for (011) indentations. This assessment becomes more complex for (111) indentations because of multiple twin nucleation. To evaluate these results it is important to consider that in addition to the critical shear stress a strong hydrostatic field is induced underneath the surface, reaching $18 \mathrm{GPa}$ for (001) surfaces and $13 \mathrm{GPa}$ for (011) and (111) surfaces at the onset of defect nucleation. Given the proximity of the above critical shear stress levels for the nucleation of the different planar defects, hydrostatic pressure is found to play a crucial role in governing defect nucleation. For instance, it is noted that while SFs are triggered for (001) indentation, the instantaneous critical shear stress induced in the most active $\{112\}<11-1>$ slip systems already exceeded the level at which twin nucleation occurred in (011) surfaces. These results indicate different sensitivities of the nucleated defects to hydrostatic pressure, so that this phenomenon is biased towards stacking fault inception in the (001) orientation because of the greater hydrostatic pressure present in that orientation. Overall by performing the FE simulations assuming specific nucleation events as a function of orientation, it was determined that the (100)-oriented crystal will preferentially twin, while for (110) stacking faults are generated prior to twinning. In the (111)-oriented sample there is a competition between twinning and stacking faults as the twinning plane (112) is close to the surface.

\section{Elevated temperature behavior}


The deformation behavior of Ta changes markedly once the critical temperature of $177^{\circ} \mathrm{C}$ is surpassed. Beyond this temperature cross-slip of screw dislocations is expected to contribute significantly to the total plastic deformation.

\subsection{Experimentally measured instrumented indentation response}

The transition from room temperature to elevated temperature can clearly be seen in the load displacement curves (Fig. 3 a-c), where at least two discrete pop-ins can be seen at any given orientation at $200^{\circ} \mathrm{C}$. Comparing the critical loads at the first pop-in event at room temperature for low loading rates $(1000 \mu \mathrm{N} / \mathrm{s})$ it becomes obvious that the (111) orientation has the lowest pop-in load $\left(\mathrm{P}_{\text {pop-in }} \approx 219 \mu \mathrm{N}\right)$ followed by the $(110)\left(\mathrm{P}_{\text {pop-in }} \approx\right.$ $255 \mu \mathrm{N})$ and $(100)$ orientation $\left(\mathrm{P}_{\text {pop-in }} \approx 307 \mu \mathrm{N}\right)$ (Fig. 4 a-c). The same trend was observed for the higher loading rate of $5000 \mu \mathrm{N} / \mathrm{s}$ (Fig. 4 a-c). Overall the loading rate dependence is almost non-existent for the (111) single crystal and increases successively over (110) to (100), where it is the most pronounced but still not significant as discussed in a previous manuscript $[20,27]$.

For the 100 oriented Ta-single crystal multiple events are clearly visible while for the (111) crystal, there is one event at low loads and a second one at higher loads (Fig 3c), both with significant pop-in displacements in the 10s of nanometer range. So even though there is a nominally enhanced capability for plastic deformation, there is no significant sign for it to be found in the (111)-crystal as the crystal still only shows few relatively large pop-ins. The transition is somewhat clearer in the (110) surface as compared to the (111) surface (Fig 3a), where at elevated temperatures above the critical temperature several pop-ins at various loads are observed. This behavior mimics the serrated flow 
characteristic for FCC-metals at both low and high temperatures. The effect of temperature on the flow behavior is most pronounced for the (100) orientation (Fig 4a), where the load-displacement curves (Fig. 3a) show a large number of relatively small displacement bursts. It should be noted that this serrated flow behavior was not observed at $100{ }^{\circ} \mathrm{C}[27]$.

In order to further understand the pop-in behavior, the cumulative distributions of the critical load for 100 indents at each condition are presented in Figures 4a-c. It is observed that the pop-in load decreases as expected with increasing temperature. This effect is most pronounced for (100) and (110) crystals (Fig 4a and b). For the (111) orientation (Fig 4c), the temperature dependence of the critical pop-in load is weak. Overall, the trend due to loading rate for all orientations is not as predominant as the temperature effect.

A more detailed analysis of the cumulative distributions was carried out to determine if changes in the incipient plasticity could be related to the activation volume the model by Mason et al. [2]. Surprisingly, all activation volumes vary from around $0.9 \mathrm{~b}^{3}$ for (111) to $0.2 \mathrm{~b}^{3}$ for (110) and (100), and were found to be almost independent of temperature and strain rate. These values are significantly lower than the $10 b^{3}$ measured by Sato et al. using strain rate jump tests [41]. This difference has several reasons: First, even the best sample has surface imperfections which tend to decrease the critical load for dislocation nucleation by stress concentration. This causes broadening of the critical load distribution which, using Mason's analysis, leads to an underestimation of the activation volume [2]. Activation volume values that are as large as those reported by Sato et al. [41] would require an unrealistically sharp pop-in load distribution. Secondly as our MD-simulations 
revealed the highly compressive stresses encountered in indentation are creating shear strains that can only be accommodated by other mechanisms such as the SFs and twins [10]. As a consequence the activation volume determined by indentation in a BCCmaterial cannot be compared to the values obtained from classical strain-rate jump tests as it describes a very specific and complex deformation scenario.

\subsection{The role of surface orientation upon the high temperature serrated behavior}

An interesting finding from the experiments is that the first pop-in load plateau is immediately followed by a quasielastic loading segment response entails immediately following the first pop-in load plateau and that this behavior becomes more noticeable (spanning a wider range of applied loads) with increasing temperature. A comparison of the post pop-in behavior for different orientations at elevated temperatures shows that the quasielastic reloading is more pronounced for the (001) orientation that also shows the most pronounced "serrated behavior". When comparing the contact response from the different surfaces, it also becomes apparent that the (001) orientation, exhibiting the greatest penetration depth burst during the pop-in, also undergoes a more noticeable quasielastic reloading. For instance for a loading rate of $5000 \mu \mathrm{N} / \mathrm{s}$ the quasiselastic reloading required to trigger the next pop-in at $200{ }^{\circ} \mathrm{C}$ is approximately $250 \mu \mathrm{N}$ for $(001)$ while (110) and (111) exhibit a second pop-in after an additional reloading of about $100 \mu \mathrm{N}$ with (111) showing the least pronounced quasielastic reloading regime.

The MD simulations showed that an increase in temperature causes a transition to a dislocation-mediated plasticity mechanism. Specifically, the comparison of the MD simulations at intermediate temperatures $(300 \mathrm{~K})$ for the three orientations, show that for 
(001) surface the transition is less pronounced [10]. Hence, the experimental findings of a marked quasielastic reloading stage past the first pop-in and the more prominent serrations for the (001) surface from the experiments at $200{ }^{\circ} \mathrm{C}$ suggest that the enhanced mobility of the remnant planar defects immediately underneath the indenter leads to sudden stages of outwards propagation. In general, for the (001), (011) and (111) surfaces, the mobility of the more linear defect character present underneath the indenter is enhanced at $200{ }^{\circ} \mathrm{C}$, but judging from the less marked serrated behavior for the (011) and (111) surfaces, the net increase in mobility appears to be relatively smaller than for the (001) orientation.

\section{Conclusion}

In this paper we have shown that there is a significant change in the deformation behavior of a high-melting point BCC-material (Ta) once the critical temperature is surpassed. The mean pop-in load increases from (111) to (110) and (100) which shows the highest resistance against localized yielding. A tentative explanation for the differences in the pop-in load of the three different orientations was found based on the stresses determined from FEM-modeling that showed that the high hydrostatic pressures encountered in indentation aid nucleating defects such as twins and SF.

At $200{ }^{\circ} \mathrm{C}$ the (100)-oriented crystal shows a highly serrated P-h-curve. The serrated flow behavior as found at $200^{\circ} \mathrm{C}$ is an evolution of the defect networks due to the specific quasi-elastic reloading at a given orientation [10].

Analysis of the cumulative distributions of the critical load for all crystals orientations showed a strong effect due to temperature while the strain rate sensitivity was found to be minimal. Fluctuations and inconsistencies in the cumulative distributions were attributed 
to surface imperfections and additional defect mechanisms besides regular heterogeneous dislocation nucleation. MD-simulations revealed the presence of higher energy mechanisms such as twinning and stacking fault generations which means that the MDsimulations are the upper-bound of the pop-in distribution [10] while the experiments in this study present the lower-bound of the distribution. Furthermore, the secondary popins were found to be a release mechanism of the stress accumulated in the quasi-elastic reloading after the initial yielding.

\section{Acknowledgements}

Parts of his work were performed under the auspices of the U. S. Department of Energy at Lawrence Livermore National Laboratory under Contract DE-AC52-07NA27344. Financial support for the work at UPC was provided by Ministerio de Educación y Ciencia (Grant MAT2011-- $\square 23375)$.

References:

[1] A.C. Lund, A.M. Hodge, C.A. Schuh, Applied Physics Letters, 85 (2004) 1362-1364.

[2] J.K. Mason, A.C. Lund, C.A. Schuh, Physical Review B, 73 (2006) 054102.

[3] C.E. Packard, O. Franke, E.R. Homer, C.A. Schuh, Journal of Materials Research, 25 (2010) 2251-2263. 
[4] C.A. Schuh, T.G. Nieh, Acta Materialia, 51 (2003) 87-99.

[5] A. Barnoush, Acta Materialia, 60 (2012) 1268-1277.

[6] N. Gane, F.P. Bowden, Journal of Applied Physics, 39 (1968) 1432-1435.

[7] D.F. Bahr, S.L. Jennerjohn, D.J. Morris, Jom-J Min Met Mat S, 61 (2009) 56-60.

[8] S. Akarapu, H.M. Zbib, D.F. Bahr, International Journal of Plasticity, 26 (2010) 239-257.

[9] A.A. Zbib, D.F. Bahr, Metall. Mater. Trans. A-Phys. Metall. Mater. Sci., 38A (2007) 2249-

2255.

[10] J. Alcala, R. Dalmau, O. Franke, M. Biener, J. Biener, A. Hodge, Physical Review Letters, 109 (2012).

[11] A.B. Mann, J.B. Pethica, MRS Online Proceedings Library, 436 (1996) null-null.

[12] Z. Wang, H. Bei, E.P. George, G.M. Pharr, Scripta Mater, 65 (2011) 469-472.

[13] S.A.S. Asif, J.B. Pethica, MRS Online Proceedings Library, 436 (1996) null-null.

[14] D.F. Bahr, J.C. Nelson, N.I. Tymiak, W.W. Gerberich, Journal of Materials Research, 12

(1997) 3345-3353.

[15] W.W. Gerberich, J.C. Nelson, E.T. Lilleodden, P. Anderson, J.T. Wyrobek, Acta Materialia, 44 (1996) 3585-3598.

[16] M. Goeken, M. Kempf, Zeitschrift fuer Metallkunde/Materials Research and Advanced Techniques, 92 (2001) 1061-1067.

[17] C. Begau, A. Hartmaier, E.P. George, G.M. Pharr, Acta Materialia, 59 (2011) 934-942.

[18] H. Bei, Y.F. Gao, S. Shim, E.P. George, G.M. Pharr, Physical Review B - Condensed Matter and Materials Physics, 77 (2008).

[19] D.-S. Xu, J.-P. Chang, J. Li, R. Yang, D. Li, S. Yip, Mat Sci Eng a-Struct, 387-38 ( 2004)

840-844.

[20] M.M. Biener, J. Biener, A.M. Hodge, A.V. Hamza, Physical Review B, 76 (2007) 165422.

[21] P. Engels, A. Ma, A. Hartmaier, International Journal of Plasticity.

[22] M.J. Cordill, N.R. Moody, W.W. Gerberich, International Journal of Plasticity, 25 (2009)

281-301.

[23] S. Brinckmann, J.-Y. Kim, J.R. Greer, Physical Review Letters, 100 (2008).

[24] J.R. Greer, C.R. Weinberger, W. Cai, Materials Science and Engineering: A, 493 (2008)

21-25.

[25] J.-Y. Kim, D. Jong, J.R. Greer, Acta Materialia, 58 (2010) 2355-2363.

[26] S.W. Lee, W.D. Nix, Philos. Mag., 92 (2012) 1238-1260.

[27] K.V. Rajulapati, M.M. Biener, J. Biener, A.M. Hodge, Philos. Mag. Lett., 90 (2010) 35-42.

[28] A.S. Schneider, B.G. Clark, C.P. Frick, P.A. Gruber, E. Arzt, Mater. Sci. Eng. A-Struct. Mater. Prop. Microstruct. Process., 508 (2009) 241-246.

[29] A.S. Schneider, C.P. Frick, B.G. Clark, P.A. Gruber, E. Arzt, Mater. Sci. Eng. A-Struct. Mater. Prop. Microstruct. Process., 528 (2011) 1540-1547.

[30] J. Li, K.J. Van Vliet, T. Zhu, S. Yip, S. Suresh, Nature, 418 (2002) 307-310.

[31] J.A. MORIARTY, V. VITEK, V.V. BULATOV, S. YIP, Journal of Computer-Aided Materials

Design, 9 (2002) 99-132.

[32] C.S. Deo, D.J. Srolovitz, W. Cai, V.V. Bulatov, Journal of the Mechanics and Physics of

solids 53 (2005) 1223-1247.

[33] J.E. Dorn, Mukherje.Ak, Transactions of the Metallurgical Society of Aime, 245 (1969)

1493-\&.

[34] S. NEMAT-NASSER, T. OKINAKA, L. NI, J. Mech. Phys. Solids, 46 (1998) 1009-1038.

[35] S. Naka, A. Lasalmonie, P. Costa, L.P. Kubin, Philosophical Magazine A, 57 (1988) 717-

740.

[36] G. Wang, A. Strachan, T. Cagin, W.A. Goddard, PHYSICAL REVIEW B, 67 (2003) 140101.

[37] J. MARIAN, W. CAI, V.V. BULATOV, Nat Mater, 3 (2004) 158-163.

[38] Z.C. Duan, A.M. Hodge, Jom-J Min Met Mat S, 61 (2009) 32-36. 
[39] Z.-L. Liu, X.-L. Zhang, L.-C. Cai, X.-R. Chen, Q. Wu, J. F.-- - Q., J. Phys. Chem. Solids, 69 (2008) 2833-2840.

[40] J. Alcalá, O. Casals, J. Ocenášek, Journal of the Mechanics and Physics of Solids, 56 (2008) 3277-3303.

[41] R.T. SATO, A.K. MUKHERJEE, Mater. Sci. Eng., 8 (1971) 74-82. 
Fig 1. Representative room temperature load-displacement (P-h) curves for (100), (110) and (111) Ta single crystals at a loading rate of a) $1000 \mu \mathrm{N} / \mathrm{sec}$ and b) $5000 \mu \mathrm{N} / \mathrm{sec}$. Curves are offset for viewing purposes.

Fig. 2: Stress fields at the imposed ratio between contact radius and indenter diameter, $\mathrm{a} / \mathrm{D}$, leading to defect nucleation. The approximate locations of defect nucleation are marked with arrows. Part (a) gives the isocontours of shear stress in (110) indentation for the planes and directions where the twin in Fig. 2(b) nucleates. Part (b) gives the isocontours of hydrostatic pressure associated with Part (a). Part (c) provides the isocontours of hydrostatic pressure at the onset where a (011) stacking fault nucleates in (100) indentation [10].

Fig 3: a) Representative load-displacement (P-h) curves at $200^{\circ} \mathrm{C}$ for loading rates of 1000 and $5000 \mu \mathrm{N} / \mathrm{sec}$ for three different Ta crystal orientations a) (100), b) (110) and c) (111). Curves are offset for viewing purposes.

Fig. 4: Normalized cumulative distributions of the critical load at room temperature and at $200^{\circ} \mathrm{C}$ for loading rates of 1000 and $5000 \square \mathrm{N} / \mathrm{s}$ for the three different Ta crystal orientations: a) (100), b) (110) and c) (111). 


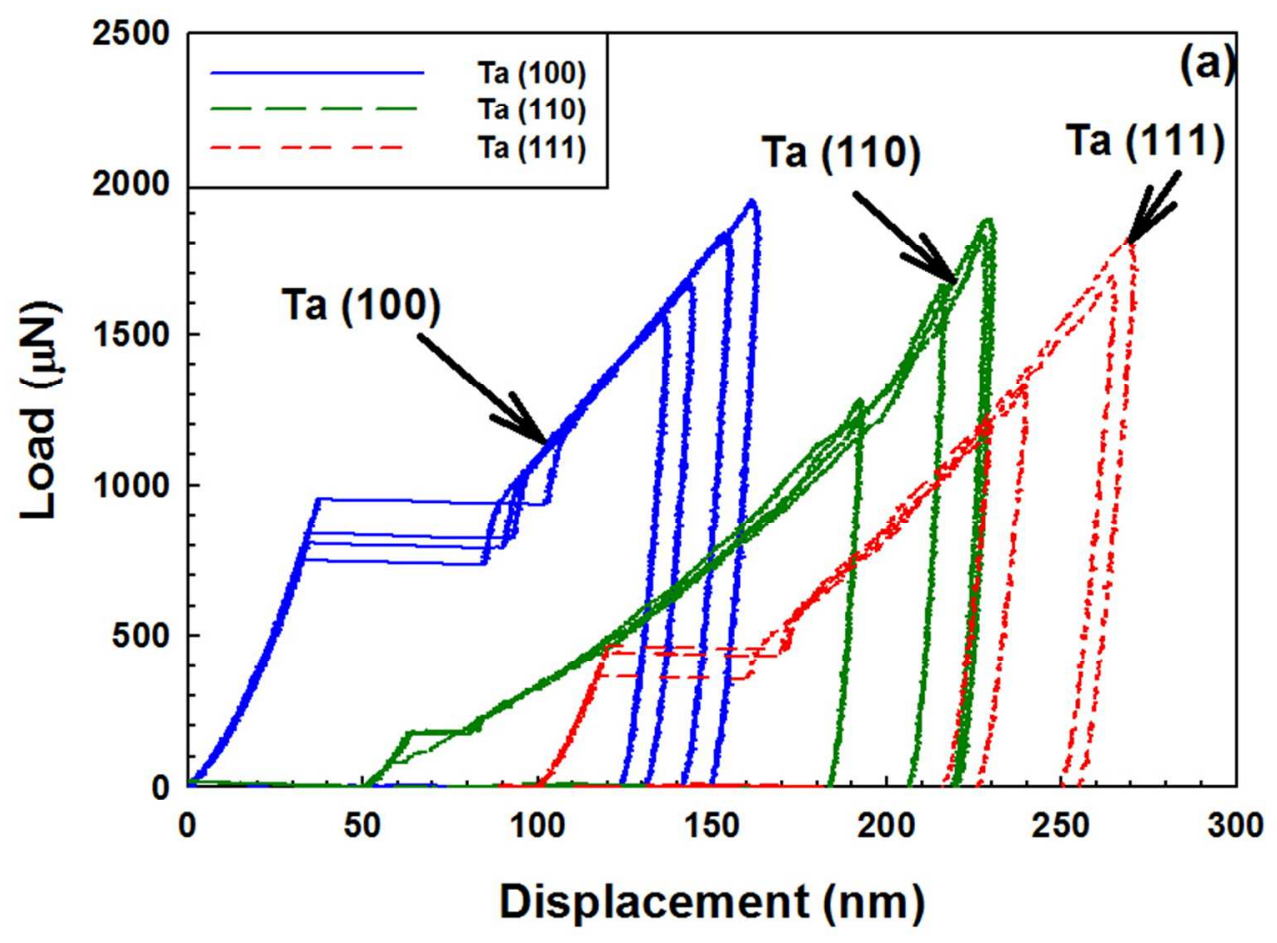

$149 \times 111 \mathrm{~mm}(150 \times 150 \mathrm{DPI})$ 


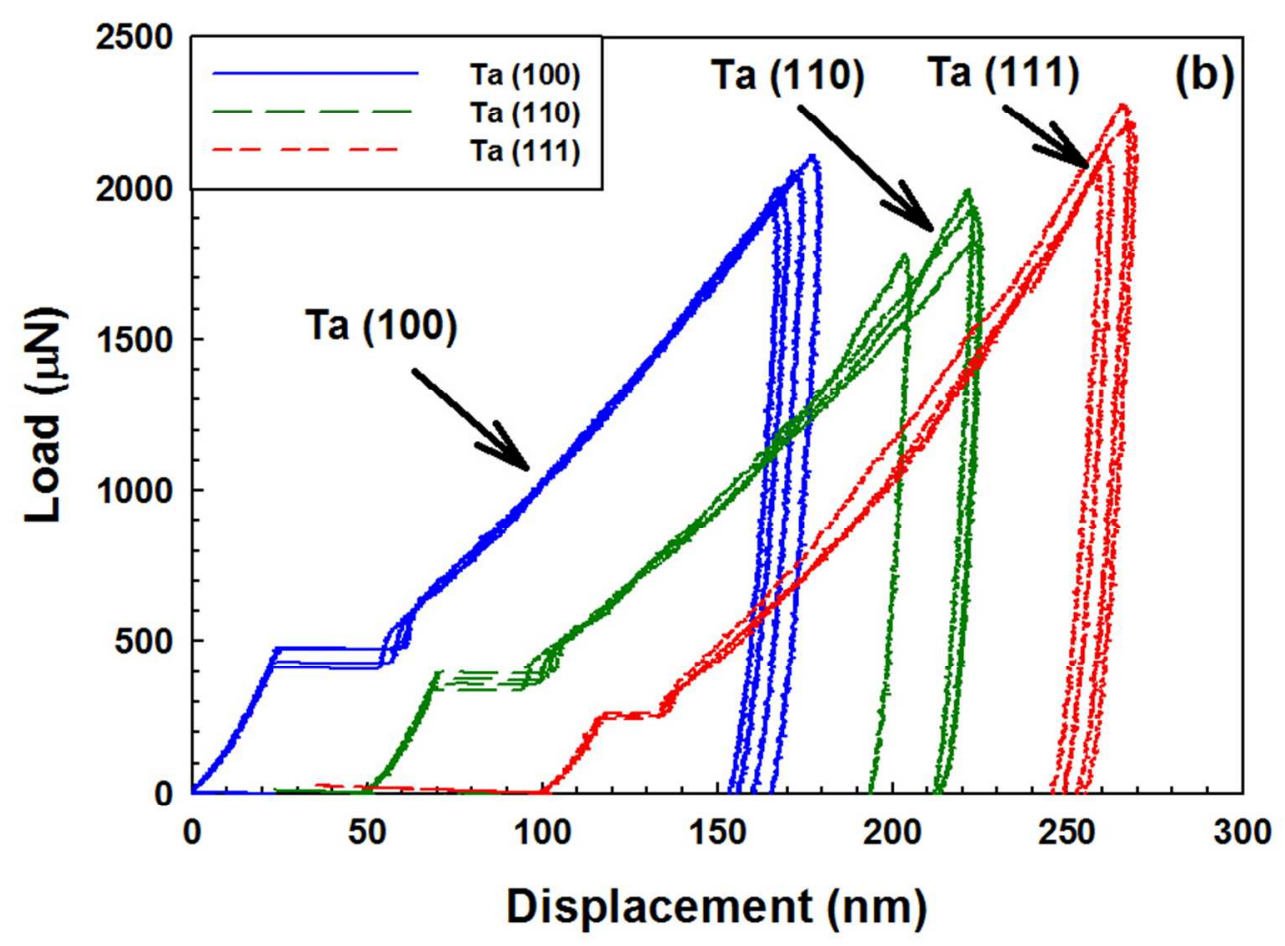

$154 \times 114 \mathrm{~mm}(150 \times 150 \mathrm{DPI})$ 


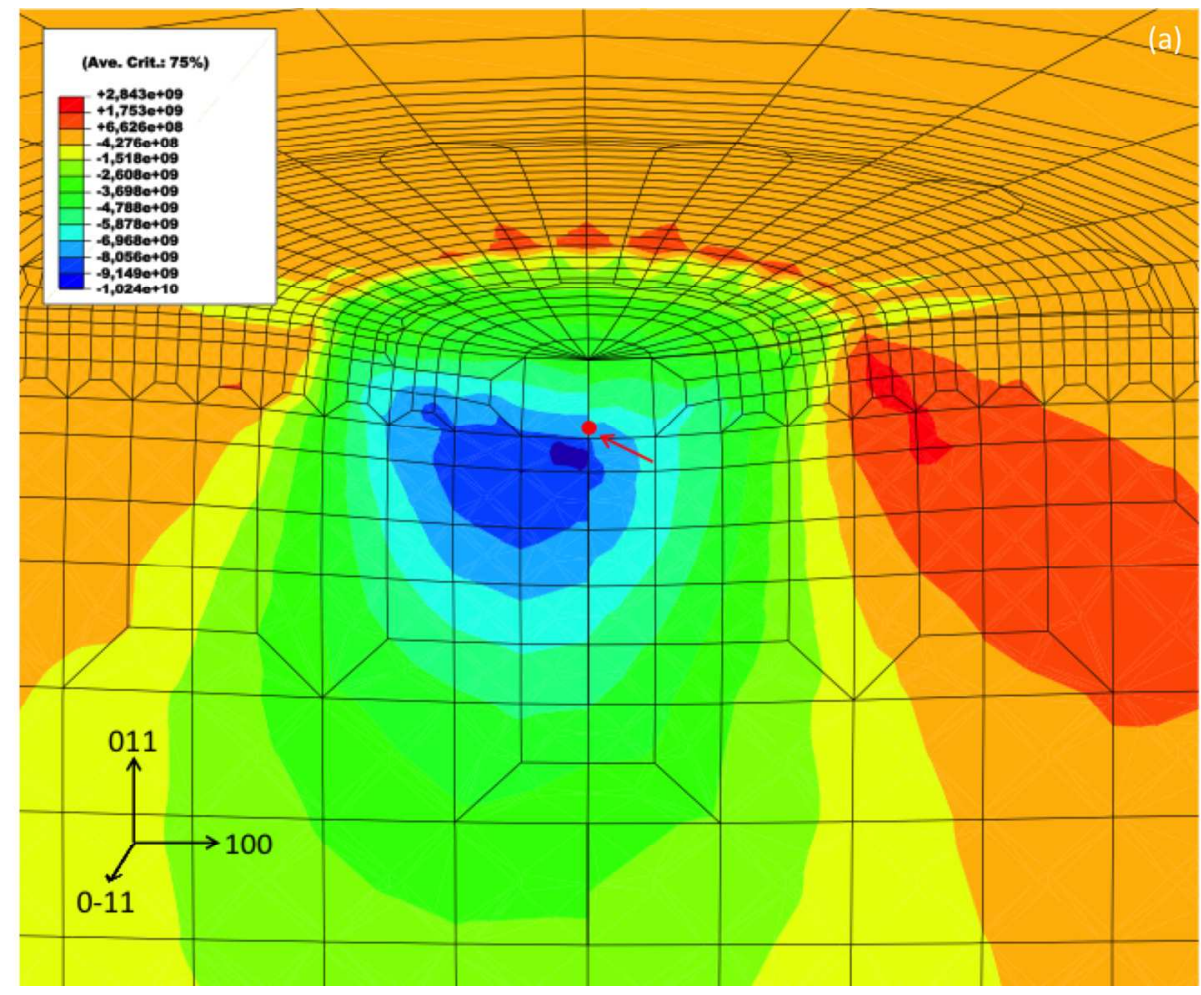

$508 \times 381 \mathrm{~mm}(72 \times 72 \mathrm{DPI})$ 


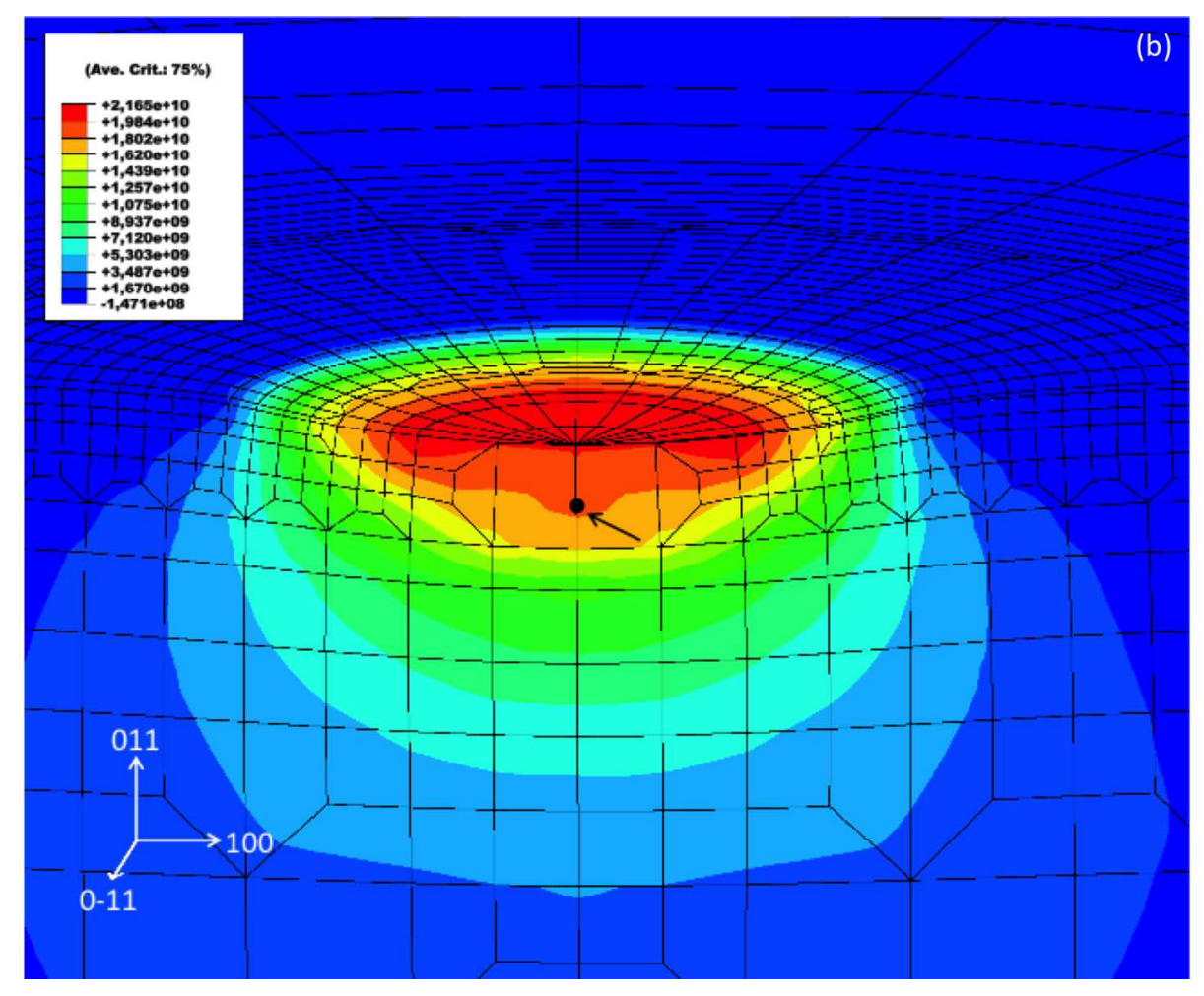

$508 \times 381 \mathrm{~mm}(72 \times 72 \mathrm{DPI})$ 


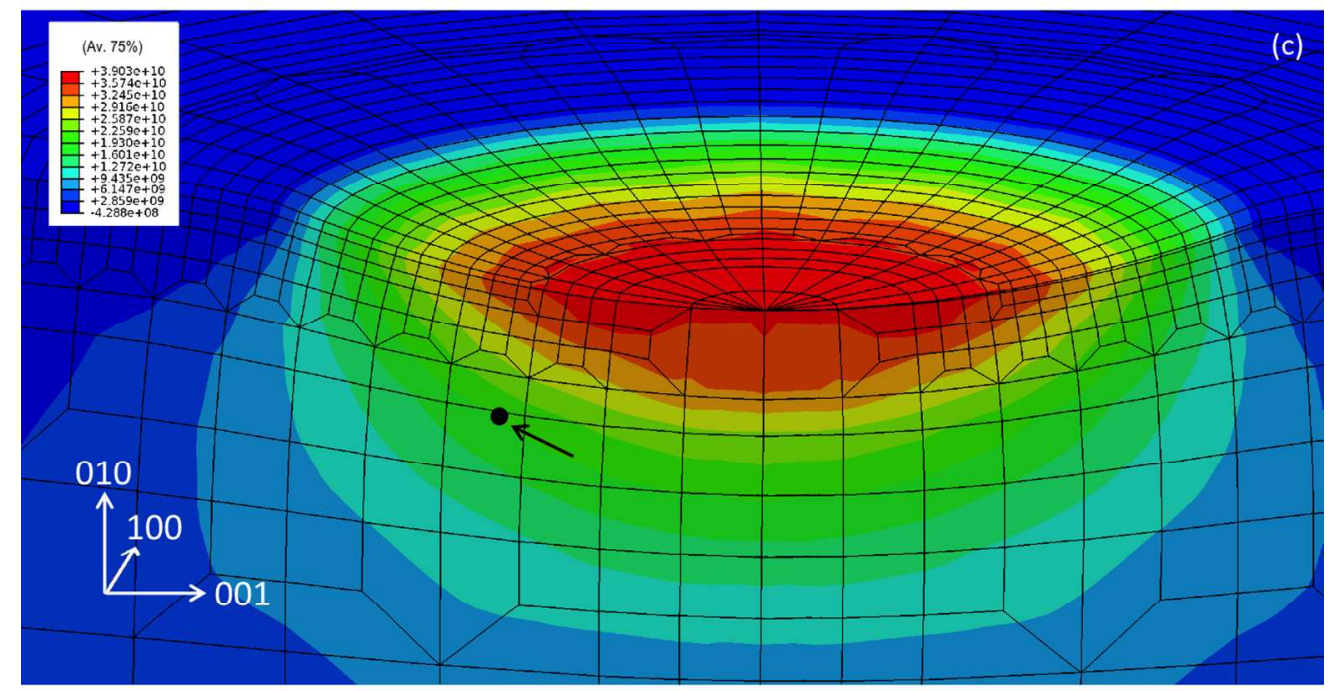

$508 \times 381 \mathrm{~mm}(72 \times 72$ DPI $)$ 


\section{Ta (100) 200C}

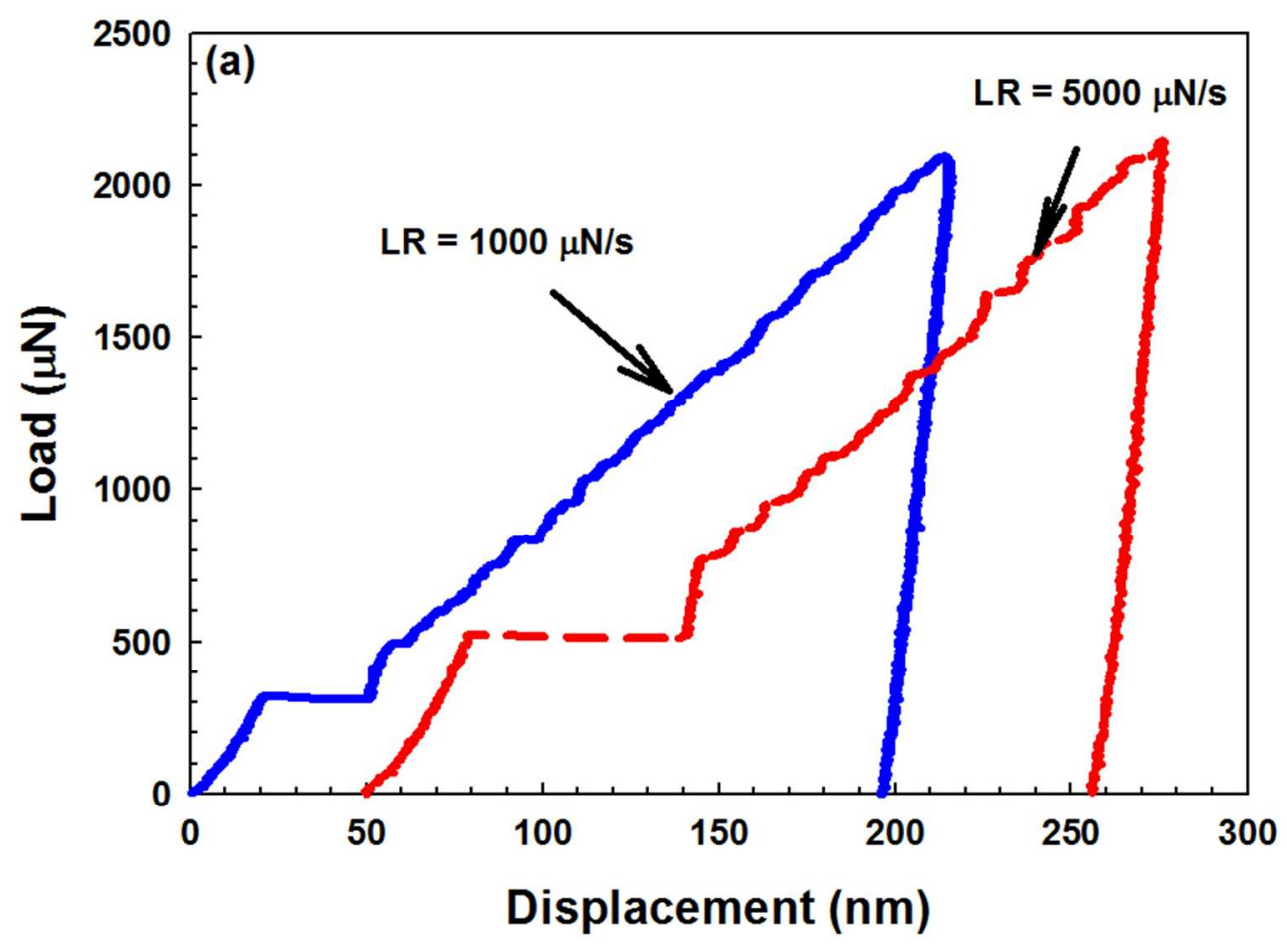

$153 \times 127 \mathrm{~mm}(150 \times 150 \mathrm{DPI})$ 


\section{Ta (110) 200C}

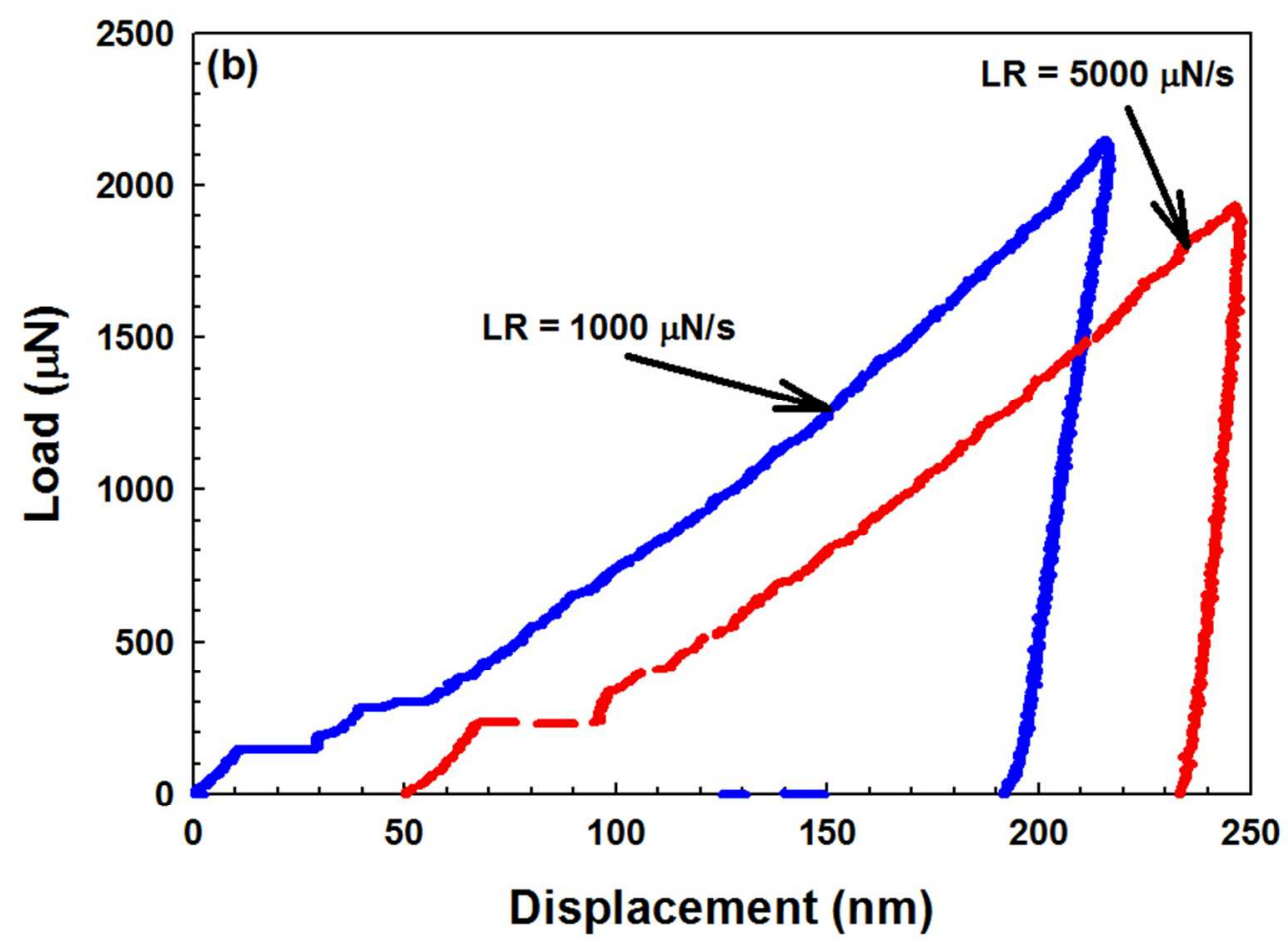

$153 \times 127 \mathrm{~mm}(150 \times 150 \mathrm{DPI})$ 


\section{Ta (111) 200C}

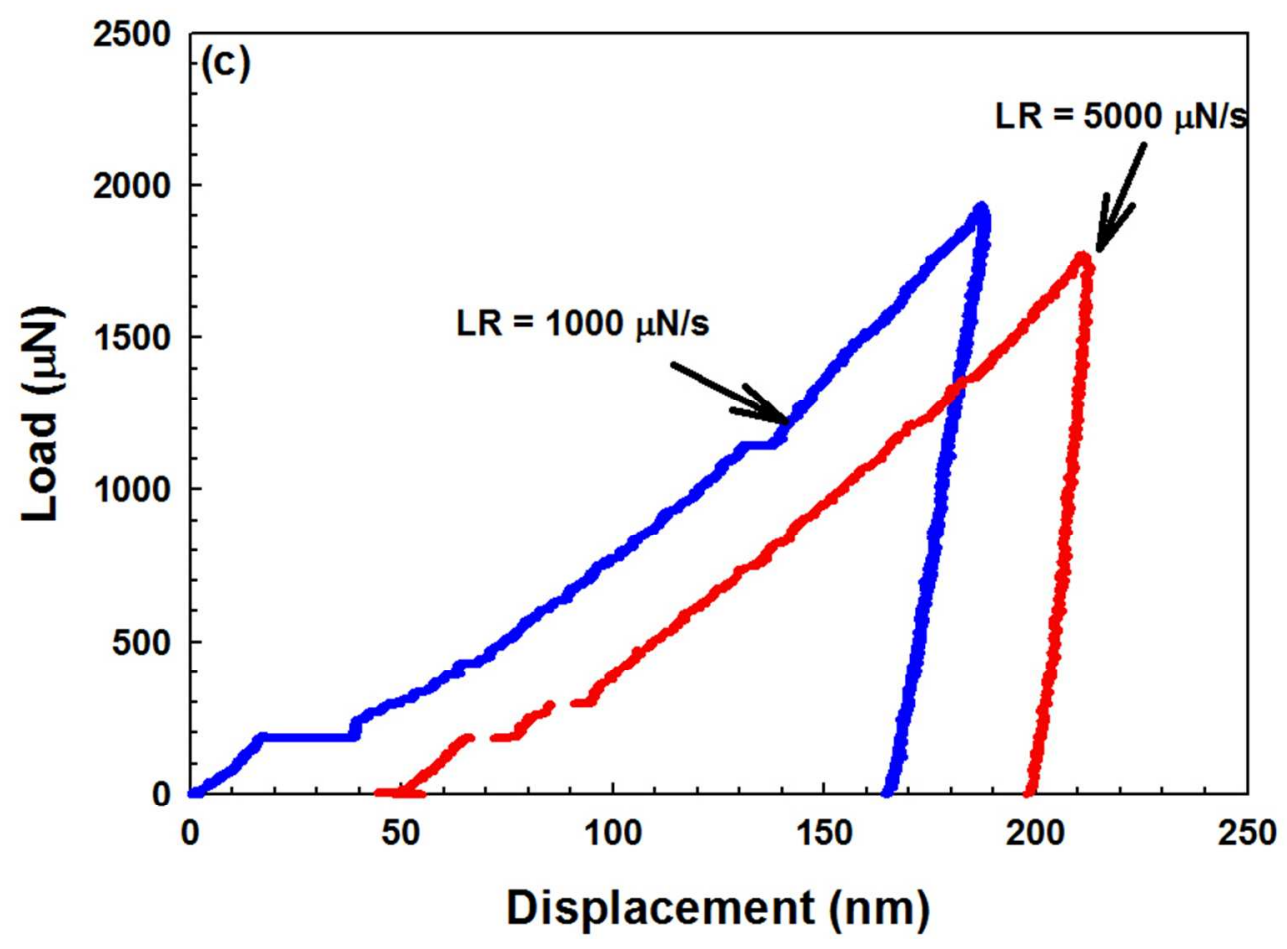

$153 \times 127 \mathrm{~mm}(150 \times 150 \mathrm{DPI})$ 


1
2
3
4
5
6
7
8
9
10
11
12
13
14
15
16
17
18
19
20
21
22
23
24
25
26
27
28
29
30
31
32
33
34
35
36
37
38
39
40
59
50
50
49
50
51
52
53
44
54

\section{$\mathrm{Ta}(100)$}

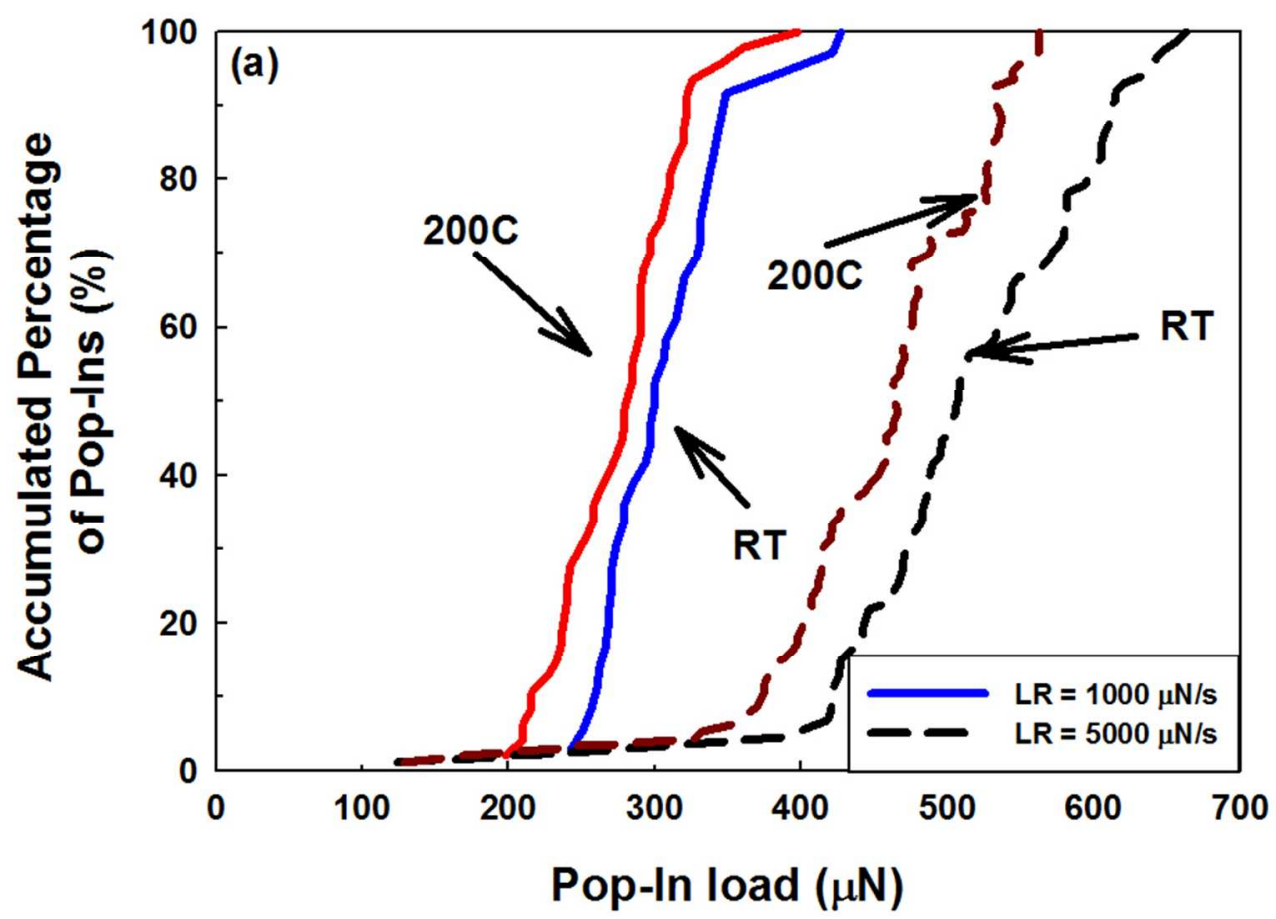

$158 \times 129 \mathrm{~mm}(150 \times 150 \mathrm{DPI})$ 


\section{$\mathrm{Ta}(110)$}

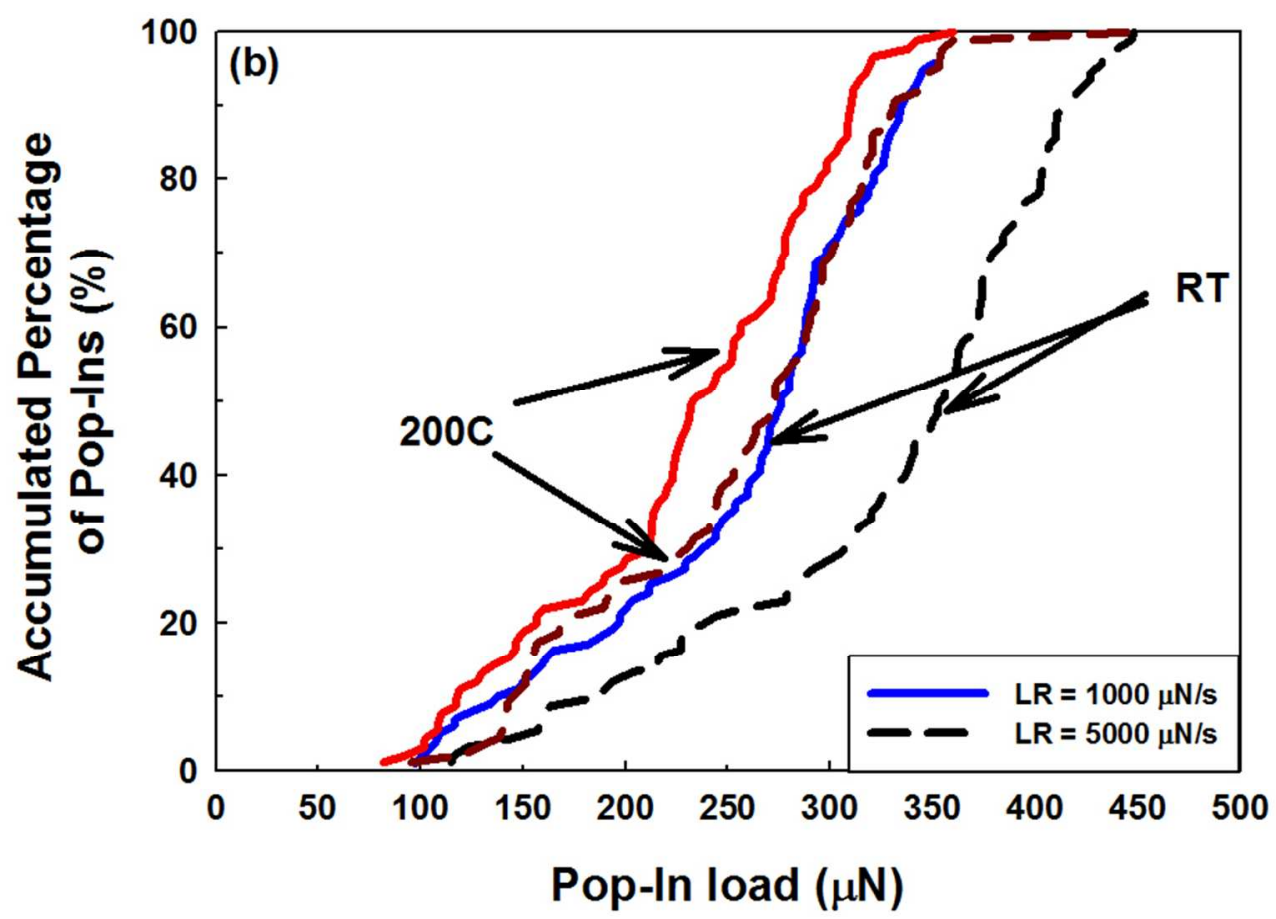

$158 \times 129 \mathrm{~mm}(150 \times 150 \mathrm{DPI})$ 


\section{$\mathrm{Ta}(111)$}

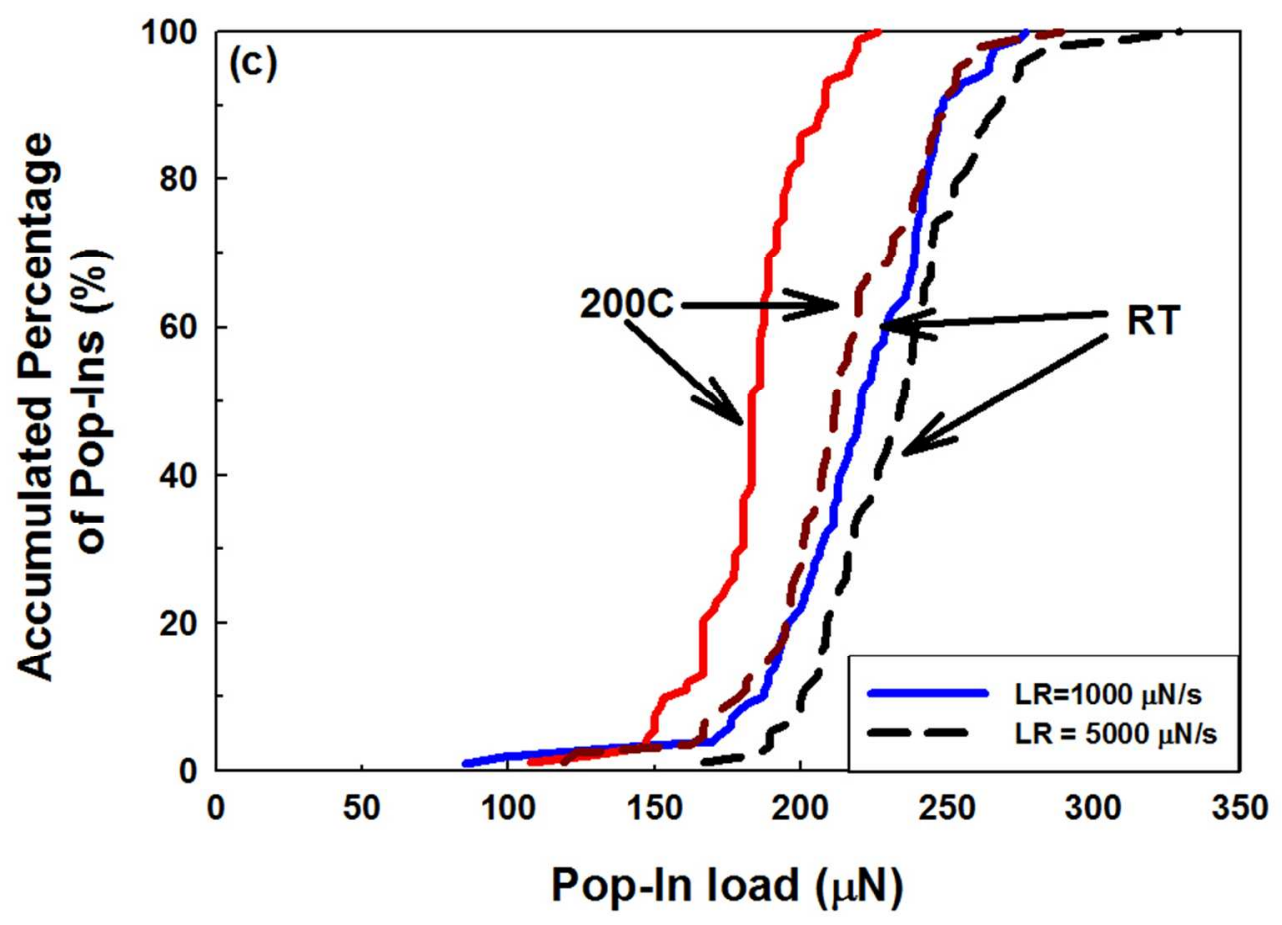

$158 \times 129 \mathrm{~mm}(150 \times 150 \mathrm{DPI})$ 